(e.g. group of mothers) and a few directly involved the participants' partner in the intervention. None used social network data to accelerate behaviour change among participants.

Conclusion Interventions often include social support from existing or created social networks as a BCT. There is a lack of interventions that purposefully utilise social network data. More qualitative research with participants and process evaluations are required. The findings of this review will be used to inform the development of a weight management intervention that mobilises social networks for behaviour change.

\section{RF26 THE EFFECTIVENESS OF PHYSICAL ACTVITIY INTERVENTIONS FOR PEOPLE WITH OSTEOARTHRITIS AND COMORBIDITY: A META-ANALYSIS OF OBESITY}

S Mckevitt* ${ }^{*}$ C Jinks, EL Healey, JG Quicke. Primary Care and Health Sciences, Keele University, Keele, UK

\subsection{6/jech-2018-SSMabstracts.114}

Background Osteoarthritis (OA) is one of the diseases with the highest prevalence of comorbidity. Clinical guidelines recommend physical activity (PA) for people with OA irrespective of comorbidity. Research investigating the effectiveness of PA interventions in OA and comorbidity is needed. Objective: To synthesise existing evidence investigating the effectiveness of PA interventions in adults with OA and obesity.

Methods A systematic review with meta-analysis was conducted (PROSPERO Registration: CRD42017055582). Six electronic databases; MEDLINE, EMBASE, AMED, CINAHL, SportDiscus and CENTRAL were searched for studies from their inception to 29.03.17. Inclusion criteria were: randomised controlled trials (RCTs) comparing the effectiveness of any PA intervention to non-PA control group; including adults aged 45 years old and over with clinical or radiographic OA at any site; at least one of the comorbidities of interest (COPD, depression, diabetes, hypertension, obesity, T2DM); and measuring pain, physical function, quality of life, global health post intervention and adverse events. Included study risk of bias (ROB) was assessed using the Cochrane risk of bias tool. Two reviewers screened titles, abstracts and full text articles, checked data extraction, and carried out ROB assessment. Random-effects model meta-analysis pooled outcomes from sufficiently homogeneous studies to calculate effect sizes (Standardized Mean Difference (SMD) with 95\% confidence interval (CI)). Meta-analysis findings of the $\mathrm{OA}$ and obesity subgroup are reported.

Results The literature search retrieved 8171 citations of which 14 studies ( $n=4224$ participants) were included in the full review, with 9 ( $n=1382$ participants) analysed in the OA and obesity subgroup. PA interventions included: aquatic, aerobic, strengthening and functional activity; of 1-18 months in duration.

Four studies of OA and obesity measuring either Western Ontario Osteoarthritis Index (WOMAC) pain, WOMAC function or Six Minute Walking Test (6 MWT) and were included in three meta-analyses. Best estimates showed PA to improve WOMAC pain ( $\mathrm{n}=3$ studies; $\mathrm{n}=547$ participants; $\mathrm{SMD}=-0.09$ (95\% CI) -0.65, 0.47), improve WOMAC function $(n=3$ studies, $\mathrm{n}=415$ participants; $\mathrm{SMD}=-0.35 \quad(95 \% \mathrm{CI})-0.89$, $0.18)$ and the 6 MWT $(n=4$ studies, $n=573$ participants;
$\mathrm{SMD}=-0.93(95 \% \mathrm{CI})-0.49,2.35)$. However, results were not statistically significant. There was substantial between-trial outcome heterogeneity $\left(\mathrm{I}^{2}=89.4 \% \quad(\mathrm{p}=0.000) ; \quad 77.5 \%\right.$ $(p=0.012) ; 97.8 \%(p=0.000)$; respectively); results should be interpreted with caution. ROB domain judgements were generally either low or unclear. A small minority of judgements were at high risk of bias.

Conclusion Best estimates suggest small beneficial effects of physical activity on WOMAC pain, WOMAC function and the 6 MWT. Mixed effectiveness among individual RCTs was likely due to heterogeneous intervention types, intensity and duration.

\section{RF27 HOW DOES CHANGING THE PLACEMENT OF FOOD PRODUCTS IN SUPERMARKETS INFLUENCE CUSTOMERS' DIETS?}

1,2J Baird*, 'SR Crozier, 'D Penn-Newman, ${ }^{1,2} \mathrm{C}$ Cooper, ${ }^{1,2} \mathrm{C}$ Vogel. ${ }^{1} \mathrm{MRC}$ Lifecourse Epidemiology Unit, University of Southampton, Southampton, UK; ${ }^{2}$ NIHR Southampton Biomedical Research Centre, University of Southampton and University Hospital Southampton NHS Foundation, Southampton, UK

\subsection{6/jech-2018-SSMabstracts. 115}

Background The dietary choices of women have a major influences on their children's diets, and childhood diet quality tracks into adulthood. Supermarkets are a major source of food for families, yet understanding of how the environment within supermarkets influences food choices is sparse. Evaluating environmental strategies to enable families, particularly those from disadvantaged backgrounds, to make healthier food choices is necessary to improve diet, reduce obesity prevalence and improve population health. We assessed the effect of improving the availability and placement of fruit and vegetables in supermarkets, and removing confectionery from checkouts, on the diet quality of women of childbearing age from disadvantaged backgrounds.

Methods This study was the pilot phase of a natural experiment with a prospective matched cluster design. The setting was a discount supermarket chain in England. The intervention had three components: i) new fresh fruit and vegetable section at store entrances, replacing smaller display at the back ii) frozen fruit and vegetables in the first aisle, and iii) removal of confectionery from checkouts. Women customers aged 18 to 45 years, with a store loyalty card, who regularly shopped at three intervention and three matched-control stores in England were recruited. Data on women's diet quality and demographic characteristics were collected via telephone survey before refurbishment, and three months after. Linear regression models adjusting for baseline diet were used to assess whether diet quality improved in women who shopped in intervention stores.

Results A total of 150 women were recruited (56 from intervention stores and 74 from control stores), and 138 provided complete data at baseline and follow-up three months after refurbishment. Participant characteristics at baseline were similar in intervention and control groups; median age was 36 years (IQR 31-41), 91\% were white British and 55\% were educated to GCSE level or below. Almost half (41\%) reported that the discount supermarket chain was their primary supermarket. Diet quality was higher among intervention than control participants at three months: $\beta=0.25$ SD $(95 \%$ CI 0.01 , 0.49 ), equivalent to four additional portions of green salad per week. This relationship weakened slightly after adjustment 
for neighbourhood deprivation, age, money spent on groceries, number of children and educational attainment $(\beta=0.23 \quad(95 \%$ CI $-0.01,0.47)$.

Conclusion Creating a healthier store layout within supermarkets may improve the diets of women customers. Future government programmes targeting the food environment could consider working with the food industry to improve product placement.

\section{RF28 INTERNATIONAL COMPARISON OF NEWS MEDIA REPRESENTATIONS OF CHILDHOOD OBESITY: DEFINITIONS, DRIVERS AND SOLUTIONS}

${ }^{1} \mathrm{C}$ Patterson*, ${ }^{1} \mathrm{~A}$ Nimegeer, ${ }^{2} \mathrm{M}$ Löf, ${ }^{3} \mathrm{M}$ Daku, ${ }^{1} \mathrm{~S}$ Hilton. ${ }^{1} \mathrm{MRC} / \mathrm{CSO}$ Social and Public Health Sciences Unit, University of Glasgow, Glasgow, UK; ${ }^{2}$ Department of Biosciences and Nutrition, Karolinska Instutet, Stockholm, Sweden; ${ }^{3}$ Department of Political Science, Texas Christian University, Fort Worth, USA

\subsection{6/jech-2018-SSMabstracts. 116}

Background Childhood obesity is a global public health concern, associated with a wide range of illnesses and economic costs. Tackling childhood obesity demands a comprehensive package of measures, targeting various levels in the whole system. However, governments often rely on individual-level solutions rather than societal-level solutions to tackle the problem. Public attention and supportiveness is often a prerequisite for political action, and news media represent a key influence on public attention to, and interpretations of, health problems and their potential solutions. This study is a comparative analysis of the framing of childhood obesity between 1996 and 2014 in national newspapers of the UK, Canada and Sweden, taking into account the specific policy contexts of each country.

Methods Quantitative content analysis of newspaper articles predominantly focused on childhood obesity, collected from the Nexis and Retriever Research databases. A total of 1839 relevant articles were coded using a framework that recorded mentions of definitions of, drivers of, and solutions to the childhood obesity problem. A random subsample of each country's articles was double-coded and inter-rater reliability was measured to ensure consistent interpretation and application of codes. Data were subjected to statistical analysis, including chi2 tests of relationships between countries and logistic regressions of changes in framing over time, with particular focus on mentions of individual- and societal-level solutions to childhood obesity.

Results Across the three countries, there was significantly $(\mathrm{p}=0.013)$ more coverage of societal-level solutions $(51.0 \%)$ than individual-level solutions (43.5\%). Between 1996 and 2014 the proportion of articles reporting on individual solutions declined $(p<0.001)$, while the proportion mentioning societal solutions remained steady. However, trends in representations of solutions differed between the three countries over time. There were also between-country differences in representations of gender, ethnocentrism, alarmism, and the extents to which diet and sedentary behaviours were associated with the problem.

Conclusion Given the influence of the media framing, an increasing focus on societal framings may be beneficial for public health advocates campaigning for political action to target the systemic drivers of childhood obesity. However, substantial differences in each country's framing of childhood obesity suggest that advocates must tailor their tactics to the dominant framing of the issue within their countries, as well as to the specific national policy landscapes in which they operate.

\section{RF29 CRITICAL DISCOURSE ANALYSIS ON THE INFLUENCE OF MEDIA COMMENTARY ON FATAL FETAL ABNORMALITY IN IRELAND}

'S Power*, 'K O'Donoghue, ${ }^{1,2}$ S Meaney. 'Pregnancy Loss Research Group, INFANT, University College Cork, Cork, Ireland; ${ }^{2}$ National Perinatal Epidemiology Centre, University College Cork, Cork, Ireland

\subsection{6/jech-2018-SSMabstracts.117}

Background Antenatal diagnosis of a fatal fetal anomaly (FFA) confronts parents with their child's mortality and creates difficult decisions including whether to terminate or continue with the pregnancy. Media offers an insight into health-related information available to the public. Readers are both active and selective in their interpretations of text, however the influential nature of media is well documented as readers are constrained by the framing of these texts. This study aimed to examine how information on FFA, termination of pregnancy for FFA (TOPFA) and perinatal palliative care (PPC) were framed in Irish published media.

Methods This qualitative study applied a critical discourse analysis, which examines the relations between discourse and social and cultural phenomena. The analysis of the text, actors, language used and its context assists in the deconstructing of text to facilitate interpretation and understanding of socially produced meanings, recognising the ability of language to generate a social world. An Irish broadsheet and online journal, both of which were easily accessible online with daily outputs and both having a daily readership in excess of 300000 were chosen for this study. An online search of FFA, PPC and TOPFA was undertaken. Language and visuals of the articles were analysed to identify the potential influence on the reader.

Results 130 articles, dated from 2012 to 2017 referencing FFA, TOPFA and PPC were identified. Themes of power and politics, international influence, ethical dilemmas and emotional appeal were embedded in the discourse, creating political influence and appealing to the emotional side of the reader to influence perceptions and views. The use of different terminology e.g. 'FFA' versus 'life-limiting condition' and the use of the word 'fetus' versus 'baby' were evident throughout the results. Representatives with different ideological standpoints chose these terms in attempts to create varying contexts and support arguments e.g. those from pro-choice groups used 'FFA' to convey the lethal prognosis and those who were pro-life used 'baby' to personify the individual who is being terminated.

Conclusion Connotations and intentional terminology were evident within the articles to convey a particular influence to the reader. Language is not neutral and therefore it is important to analyse the information being delivered through media to the general public. This is of additional relevance as a referendum to adapt Irish Abortion Laws is imminent which has the potential to change health service provision and requires the delivery of accurate knowledge to the public. 